\title{
Proyecto museográfico de la Sala de dibujos y estampas del Museo de Bellas Artes de Córdoba
}

\author{
Raniero Baglioni \\ Centro de Intervención del Instituto Andaluz del \\ Patrimonio Histórico \\ Fuensanta García de la Torre \\ Directora del Museo de Bellas Artes de Córdoba \\ Isidoro Guzmán Espinosa \\ Técnico de Exposiciones
}

\section{Resumen}

La importancia de la reestructuración museográfica de la sala de dibujo del Museo de Bellas Artes de Córdoba, estriba en el hecho de poder exponer al público en una sala, oportunamente modificada, con plena garantía para su conservación, la importante colección de obras gráficas y dibujos propiedad del mismo.

Gracias a los medios técnicos y científicos que tenemos a nuestra disposición se ha podido realizar una sala que cumpla tanto con las normas de conservación preventiva, como con los fines museológicos y museográficos.

De hecho conservar no significa descontextualizar las obras de arte, sino buscar soluciones lógicas a problemas complejos.
La organización de una exposición requiere, entre otras muchas actuaciones, el diseño de distintos elementos de montaje que contribuyan a presentar los bienes culturales expuestos en las mejores condiciones posibles de conservación, a la vez que garanticen la más completa difusión de este patrimonio. Conservar y difundir, serán pues dos objetivos básicos a cumplir en cualquier diseño de un proyecto museográfico, bien sea para una exposición temporal o bien para una permanente.

Con estos objetivos presentes, el Museo de Bellas Artes de Córdoba se planteó la modificación de los sistemas expositivos de su Sala de dibujos y estampas, teniendo en cuenta que el montaje existente en ese momento respondía a un proyecto inaugurado en 1986, por lo que el envejecimiento natural de los materiales junto a los avances llevados a cabo por las ciencias y técnicas auxiliares de la museografía hacían evidente la necesidad de sustitución de los elementos expositivos.

Así, se planteó ante la Dirección General de Instituciones del Patrimonio Histórico que, a través del Instituto Andaluz del Patrimonio Histórico (IAPH), aprobó y financió las reformas necesarias para llevar a cabo un nuevo Proyecto museográfico de la Sala de dibujos y estampas del Museo de Bellas Artes de Córdoba, redactado por Isidoro Guzmán y Raniero Baglioni bajo la coordinación de Fuensanta García y la colaboración de José Ma Palencia y Alfonso Blanco, Asesor Técnico de Conservación e Investigación y Restaurador del Museo de Bellas Artes de Córdoba, respectivamente.

Una vez remodelada, la sala fue inaugurada en marzo de 200 I junto a las de Arte medieval, renacentista y manierista en Córdoba, cuya museografía también fue renovada.

\section{LAS COLECCIONES DE DIBUJOS Y ESTAMPAS}

La Colección de dibujos y estampas del museo cordobés es digna de consideración tanto por la cantidad de obras que la componen como por la singularidad de muchas de ellas!.
Museo / Conservación / lluminación / Exposición / Museo de Bellas Artes / Córdoba 
$\longleftarrow$
$\square$
El origen de la colección de dibujos está en el 20 de junio de 1877, cuando se adquiere "una colección de dibujos autógrafos de pintores antiguos notables, cordobeses y nacionales para el Museo de la Provincia"2, por la que se paga a un casi desconocido Matías Sanz un total de 300,75 pesetas. Aquí tenemos el germen de la que actualmente hemos de considerar como una interesante colección de dibujos dentro del conjunto de los museos españoles. A partir de esa lejana fecha de 1877 y hasta 2001 , numerosos son los dibujos que incrementaron aquel primer conjunto adquirido a Matías Sanz y que sin lugar a dudas procedía de la importante colección de José Saló, pintor catalán afincado en Córdoba, donde desempeñó numerosos cargos públicos, entre otros el de director del propio museo. Este incremento, producido por los tradicionales sistemas de adquisición, depósito o donación, culmina de momento en 200 I, en que la Consejería de Cultura de la Junta de Andalucía deposita en este Museo de Bellas Artes de Córdoba3a un singular dibujo de Alonso Cano (Granada, I60 I- 1667), Proyecto de fuente, adquirido dentro de las actividades propuestas por el museo para celebrar el IV Centenario del nacimiento del maestro granadino.

Forman estos fondos dibujos ejecutados por maestros de los siglos $\mathrm{XVI}$ al $\mathrm{XX}$, procedentes fundamentalmente de escuelas españolas con claro predominio de los maestros cordobeses, sin faltar algún representante de las escuelas inglesa, italiana y francesa.

En cuanto a la colección de grabados y estampas, hemos de remontarnos a 1903 cuando siendo director del museo Enrique Romero de Torres se crea la denominada "sección de arte moderno". A partir de ese mismo momento se comienza la colección de grabados que estaría constituida por obras, al igual que los dibujos, procedentes de adquisiciones, depósitos y donaciones, siendo las últimas obras ingresadas varias series que han sido depositadas por la Junta de Andalucía. Hemos de datar la colección entre los siglos XVII y XX, considerándola numéricamente casi equiparada a la de dibujos, pero con un interés y una calidad por debajo de ella como conjunto, aunque hay interesantes ejemplos de series o estampas de diferentes escuelas europeas, predominando la representación de grabadores españoles.

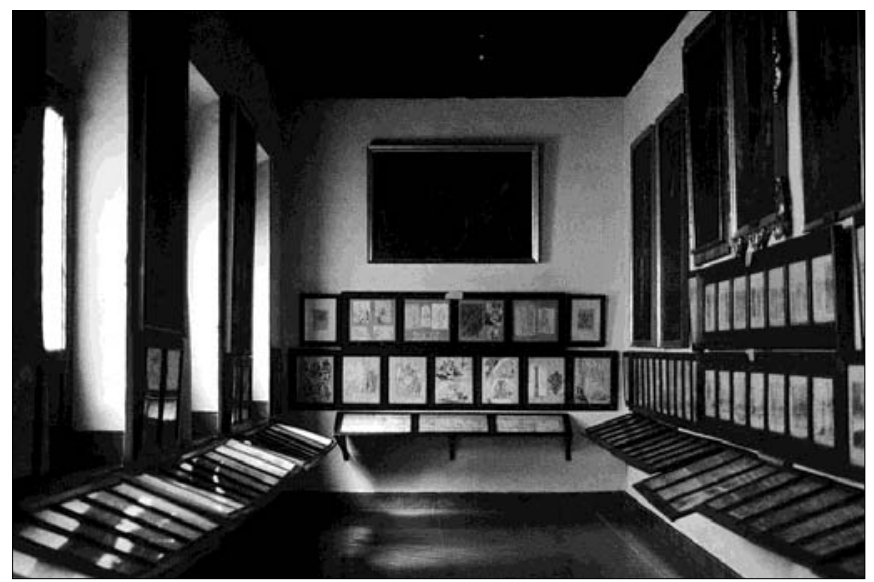

PROGRAMA MUSEOLÓGICO Y PROYECTO MUSEOGRÁFICO DE LAS COLECCIONES DE DIBUJOS Y ESTAMPAS.

Gracias a la documentación, tanto escrita como gráfica, conservada en el museo se ha podido ir reconstruyendo parte de los que hoy denominamos programa museológico y proyecto museográfico de estas colecciones desde el siglo XIX, aunque desafortunadamente restan por conocer muchos aspectos de cómo estos se materializaron.

Nos queda aún en el terreno de la hipótesis el programa museológico, pues conocemos escasísimos datos sobre la valoración y los criterios que guiaron a los responsables del museo entre el siglo XIX y I98I con respecto a estas colecciones, aunque sí sabemos que tenían un especial interés por su incremento, más en el caso de los dibujos que de los grabados y que prácticamente todos los fondos de dibujos y algunos de los grabados estaban expuestos. Actualmente centramos el interés de la exposición de las diferentes secciones en aquellos maestros cordobeses o que han desarrollado parte de su producción en la ciudad y que forman parte de la colección estable y los depósitos. Cambia esta intención con respecto a las obras sobre papel, cuya exposición no queda circunscrita a la escuela cordobesa por sus especiales características.

Algo más conocemos sobre el proyecto museográfico, aunque difícil se nos hace denominar como tal la intención de los directores, conservadores o restauradores del museo, que durante mas de un siglo fueron diferentes miembros de la Familia Romero de Torres.

Por razones muy diversas conocemos bastantes más datos sobre los dibujos que sobre los grabados. Reseñaremos aquí los hitos fundamentales de la museografía de estas colecciones desde el siglo XIX hasta el Proyecto que nos ocupa 4 .

\section{Desde el siglo XIX a I98 I}

Como se ha dicho, por un mejor conocimiento de los dibujos nos centraremos básicamente en ellos, aunque bastantes de las situaciones que vamos a exponer son extensivas al resto de estos fondos sobre papel: coincidencia en sistemas de montaje, mismas colecciones de origen -Avilés, Camacho Padilla, Romero de Torres o Bea Pelayo-, mismas salas y condiciones de exposición, etc.

En el caso de los dibujos debieron exponerse ya en el siglo XIX, pues se puede deducir esto de referencias bibliográficas como la de Ramírez de Arellano en 1896 o fotográficas, tal es el caso de una interesante foto de Laurent, sin fecha pero anterior a 1896, en las que en la capilla, adaptada a sala de exposiciones, hay unas vitrinas en las que parece que se exponen dibujos. El 18 de agosto de 1902 el conservador del museo pide "trasladar a otra sala que reúna mejores condiciones que la en que están instalados en la actualidad la notable colección de dibujos de dicho museo", 
dato que constata la temprana exposición de la colección. Veinte años después, en 1922, Félix Boix menciona como interesantes colecciones de dibujos expuestas al público la de la Academia de San Carlos de Valencia (hoy en Museo de Bellas Artes) y la del Museo de Bellas Artes de Córdoba.

Las referencias se van completando con datos aportados por el archivo, en el que constan repetidas facturas de adquisición de materiales para montaje de dibujos con pormenorizados detalles de precios y proveedores, pero escasas referencias a otros datos que nos hubieran resultado hoy de gran interés para hacer la historia de éstas colecciones y de su museografía.

Llegamos así a 1927, en que se instala la denominada Galería de dibujos, en un espacio que hasta entonces había estado ocupado por pinturas. Desconocemos la motivación de ésta determinante decisión que sin duda, y pese a los inconvenientes de conservación que supuso con el paso del tiempo, contribuyó a su difusión y a que se publicaran algunos de ellos por los máximos especialistas de la época en dibujo español como Félix Boix, August L. Mayer, Francisco Javier Sánchez Cantón o Diego Angulo.

Este montaje con escasísimas modificaciones se mantuvo hasta 1981. Aquí se expusieron los denominados dibujos antiguos del siglo XVI al XVIII y algunos ejemplos posteriores. Estaban montados sobre marcos protegidos por cristales, instalados a modo de telarillos o pupitres, con iluminación natural que entraba por las numerosas ventanas de la sala protegidas por cortinas que ejercían una ligera acción filtrante del sol, debiéndose señalar que no hubo luz eléctrica en el museo hasta la reforma de 1981-1986.

Esta instalación fue sufriendo un progresivo deterioro a partir del fallecimiento de Enrique Romero en 1956. hasta llegar a la grave situación en que se encontraba en 1981: las cortinas habían sido eliminadas, el agua de lluvia entraba por las desvencijadas ventanas y abundantes manchas de humedad y cola habían ido apareciendo en los dibujos, lo que junto a la oxidación natural de las tintas ferrogálicas de muchos dibujos, daban un aspecto nada recomendable a una colección que por méritos propios y el esfuerzo de varias generaciones debe considerarse el conjunto de fondos más importante del museo junto a los de pintura cordobesa a partir del siglo XIV. Paralelamente a éste aspecto visible de deterioro, la sorpresa se acentuó en el desmontaje efectuado a fines de 1981, al observar que el perfil de algunos dibujos había sido íntegramente "transferido", por la acción de la luz, al soporte sobre el que se habían pegado varias décadas atrás.

No hay en los grabados un espacio específico de exposición como en los dibujos. Eran muchos menos los expuestos ya que la mayoría de los mismos se encontraban guardados en un gran cajón de madera junto a los últimos dibujos ingresados en el museo que ya no se expusieron e incluso en algunos casos ni siquiera se inventariaron.
Los que se exponían lo estaban de manera aislada en las diferentes salas en las que por su cronología o colección de origen les correspondían. En éstas salas: Arte Moderno, Angel Avilés, Bea Pelayo o Mateo Inurria, se utilizaban los mismos criterios y montajes para dibujos, grabados, acuarelas y pinturas sobre papel: marcos individuales de madera pintados en marrón oscuro y protegidos por cristal o los marcos de muy diversos tipos y formatos que traían de sus colecciones de origen. La iluminación en estas salas seguía siendo la natural, pero en general las obras estaban menos afectadas por los efectos nocivos de la luz del sol por estar expuestos más alejados de las ventanas.

Con respecto a los dibujos y grabados que forman parte de la Colección Romero de Torres, cabe decir que hasta 1991 en que se incorpora al museo -al que siempre estuvo especialmente vinculada y en la que se encontraron dibujos y grabados incluidos en sus inventarios- se mantuvieron en la anexa vivienda familiar, donde tenían un carácter de patrimonio privado que nada tenía que ver con los criterios de un museo, manteniéndose como elementos decorativos en distintas dependencias o guardados en carpetas y cajones junto con diferente tipo de documentación.

Interesante resulta la cita de Gaya Nuño en 1981, reiterando lo publicado por él mismo en 1968, al resaltar la importancia de la colección de dibujos y señalar que "ésta es materia [los dibujos] prácticamente prohibida en España, dado el misterio con que los museos ocultan sus colecciones de dibujos de maestros antiguos. Excepción honrosa es el Museo Provincial de Bellas Artes de Córdoba"s.

Sin duda, el hecho de haber estado expuestos de manera continua durante décadas y en las condiciones señaladas, ha dado una merecida fama a la colección y ha significado un importante hito en su difusión, pero ha contribuido de manera inexorable a su deterioro.

\section{Actuaciones entre 1981 y 2000}

El freno al proceso imparable de daño se puso en 1981, año en que con motivo de unas obras de reforma general del edificio del museo y de restaura-

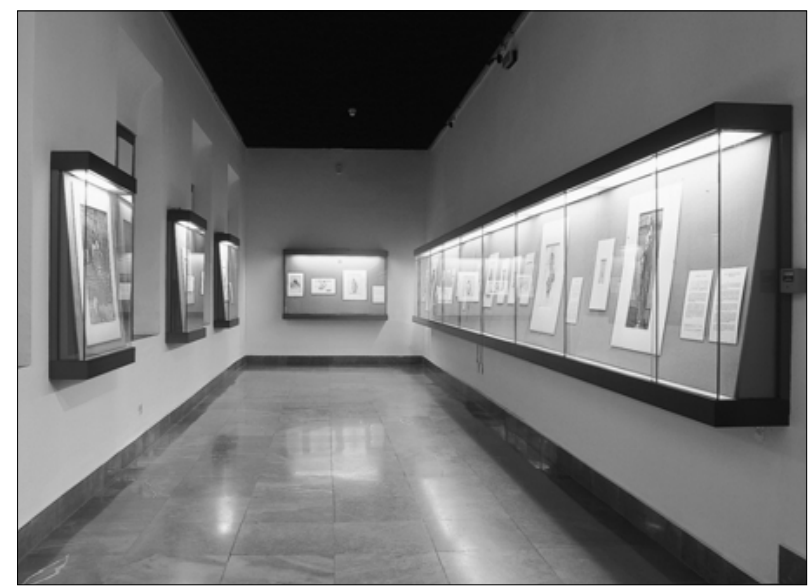


ción de sus colecciones, se acometió el desmontaje de las salas de exposiciones, iniciándose al año siguiente la restauración de dibujos, grabados, carteles y pinturas sobre papel en el actual Instituto del Patrimonio Histórico Español, proceso de restauración en el que llevamos trabajando conjuntamente durante los últimos veinte años, habiéndose restaurado en el IAPH, en 200I, el último dibujo depositado por la Junta de Andalucía.

Finalizadas las obras de reforma, los criterios museológicos y museográficos cambian sustancialmente. Se decide la exposición rotativa de las colecciones de dibujos y grabados, con muestras monográficas que contribuyan, por un lado, a la conservación de los fondos y por otro, a su necesaria difusión. A partir de entonces y hasta 2000, se expusieron o bien en la Sala de exposiciones temporales o bien en la Sala de dibujos y estampas, que ocupa el mismo espacio que la Galería de dibujos instalada en 1927.

Desde 1986, se han venido celebrando muestras temporales de dibujos y grabados, con un máximo de tiempo de exposición de seis meses. Además, entre 1987 y 1994, se celebraban exposiciones temporales de fondos sobre papel externos al museo, cosa que ahora hacemos sólo con las colecciones propias.

Tras la reapertura del museo en 1986, en la Sala de exposiciones temporales, las obras sobre papel se exponían en marcos individuales, mientras que en la Sala de dibujos y estampas se mostraban estas colecciones en unas vitrinas de pared, de metal, con un fondo tapizado en tela y un frente de cristal con un filtro para eliminar la acción de los ultravioletas que emitía la luz fluorescente de su iluminación 6 . En ambos casos se llevaba un control riguroso de la iluminación, eliminando la natural y la artificial o bien era indirecta o estaba controlada por un potenciómetro que regulaba la intensidad de la misma. Los dibujos, grabados, acuarelas y pinturas sobre papel no expuestos se almacenan en planeros y en un armario de seguridad.

"Tanto los expuestos como la mayoría de los almacenados se encuentran montados sobre paspartús de ventanas, realizados con cartones de $\mathrm{pH}$ neutro, estando sujetos a ellos mediante adecuados sistemas reversibles y protegidos en su parte delantera con unas láminas de papel tipo mylar transparentes que impiden el deposito del polvo y los roces sobre la superficie de los dibujos, protegiéndolos así durante su exposición, almacenamiento y procesos de manipulación necesarios para estos actos"7.

Analizando estas vitrinas en las que se exponían dibujos y grabados, hemos de observar que estaban construidas con un armazón de perfiles de acero, sobre el cual estaba atornillada una estructura de aluminio anodizado, que sujetaba los cristales de cierre y enmascaraba el alojamiento del sistema de iluminación.

El plano inclinado sobre el cual descansan los dibujos, es un panel de melamina, forrado de corcho y tapizado en tela. Las partes posterior e inferior de la vi- trina estaban abiertas y comunicadas directamente con el medioambiente de la sala.

El sistema de iluminación estaba compuesto por un fluorescente en cada vitrina pequeña y una serie de seis tubos también fluorescente y enfilados en la vitrina grande.

La características de estos tubos eran de 18W/ 33 TLD 34 marca Philips de $60 \mathrm{~cm}$. de longitud, para las vitrinas pequeñas, y de $58 \mathrm{~W} / 33$ TDL 34 marca Philips de 1,50 m de longitud para la vitrina grande. Dichos tubos estaban montados en la tapas de cierre, por la parte superior de la vitrina conjuntamente con los transformadores y los balastros electrónicos de regulación de la intensidad lumínica. La instalación eléctrica pasaba por detrás de los expositores y los cables iban sueltos tocando o traspasando el armazón metálico sin ningún tipo de protección. El único elemento que dividía la zona de alojamiento de la fuente luminosa con el interior de la vitrina, era un vidrio opaco con un filtro de eliminación de las radiaciones ultravioletas simplemente apoyado en el armazón metálico En los cristales perimetrales de cierre de la vitrina se aplicó igualmente una película adhesiva con acción filtrante de las radiaciones ultravioletas.

Como podemos deducir de esta breve descripción de los contenedores, cuando se plantea la remodelación de la museografía de la sala, estos ya no reunían ninguna de las condiciones especificas necesarias en la actualidad para la conservación de un material tan delicado como el papel, soporte constitutivo de los bienes culturales que nos ocupan.

Para que las vitrinas existentes fueran efectivas deberían haber sido transformadas con el fin de:

I. Hacer la vitrinas estancas, para crear un microclima fácilmente controlable y adaptable a la necesidad de conservación de la colección. Para conseguir tal efecto se debería, a parte de solucionar el problema de la estanqueidad, haber previsto un sistema de alojamiento (cajones, bandejas, etc) para el material tampón necesario (gel de sílice o art-sorb) para su acondicionamiento.

2. Trasladar el sistema de iluminación fuera del espacio expositivo de la vitrina, no solo manteniéndola separada con un vidrio, ya que el calor que pueden producir los balastros y los transformadores puede entrar en el interior de la vitrina creando descompensaciones termo-higrométricas y movimientos de circulación del aire, con la relativa estratificación del mismo produciendo resecaciones puntuales, contracciones, dilataciones localizadas y tensiones innecesarias del soporte, que, a la larga, serían dañinas para los bienes en cuestión.

En este sentido, era importante tener en consideración la falta de uniformidad en la iluminancia del interior de la vitrina considerando que, los niveles eran de 84,9 lux en la zona cercana a la fuente luminosa, de 12,6 lux en la parte baja de la vitrina y de 28,6 lux 
en la zona intermedia. Esto implicaba que no tener ni una uniformidad lumínica ni tampoco una correcta lectura del documento gráfico, porque se creaban zonas de sombra o de poca visibilidad que impedían una visión correcta de los dibujos y grabados.

3. Cerrar herméticamente la parte adosada al muro y la parte inferior de las vitrinas separándolas del muro de sostén para tener una ventilación adecuada y evitar la influencia de la humedad contenida en el mismo, sobre todo en las vitrinas adosadas al muro que da al patio.

De las medidas termo higrométricas tomadas en la sala, y en los espacios detrás de las vitrinas, ya sea en la pared izquierda que comunica con el exterior, como en la derecha colindante con la biblioteca, la lectura pone en evidencia una diferencia entre una pared $y$ otra de casi un grado centígrado de temperatura y un 10\% de humedad relativa. Esto significa que existe realmente una descompensación climática, que seguramente se ve acentuada en los cambios de estaciones y entre el día y la noche influenciando directamente los cambios termo higrométricos que se pueden verificar en el interior de las vitrinas.

Ante todo esto se tomó la determinación de sustituir los elementos expositivos considerando, además:

- la dificultad de apertura de las vitrinas que afecta a la correcta manipulación y facilidad de acceso a la zona expositiva durante los montajes, y

- la fácil penetración de polvo en su interior por falta de estanqueidad y la acción negativa de este para la conservación de los bienes culturales.

\section{EI Proyecto museográfico de la Sala de Di- bujos y Estampas: 2000-200I}

La propuesta de proyecto que se realizó a raíz de la investigación previa y de la necesidad de solucionar los problemas descritos fue la siguiente:

I. Eliminar las vitrinas y presentar dibujos y grabados en un nuevo sistema expositivo autoportante separado de las paredes de la sala, para aislarlo térmica e higrométricamente de la influencia de la humedad contenida en los muros, evitando así las diferencias termo higrométricas detectadas en la fase de estudio. Se intentaba con ello garantizar que los muros del contenedor revestido de paneles de madera que actúan de material tampón, amortigüen las variaciones climáticas al máximo, considerando que si esto no fuera posible es mejor tener movimientos climáticos importantes pero lento en el tiempo, que muchos débiles y rápidos.

2. Montaje de los dibujos y los grabados y cualquier otra obra sobre papel de las colecciones del museo, en un doble marco de seguridad especialmente diseñado para la conservación de este tipo de obra, que facilita la manipulación del material original sin tocarlo, reduciendo al máximo el riesgo para su integridad.

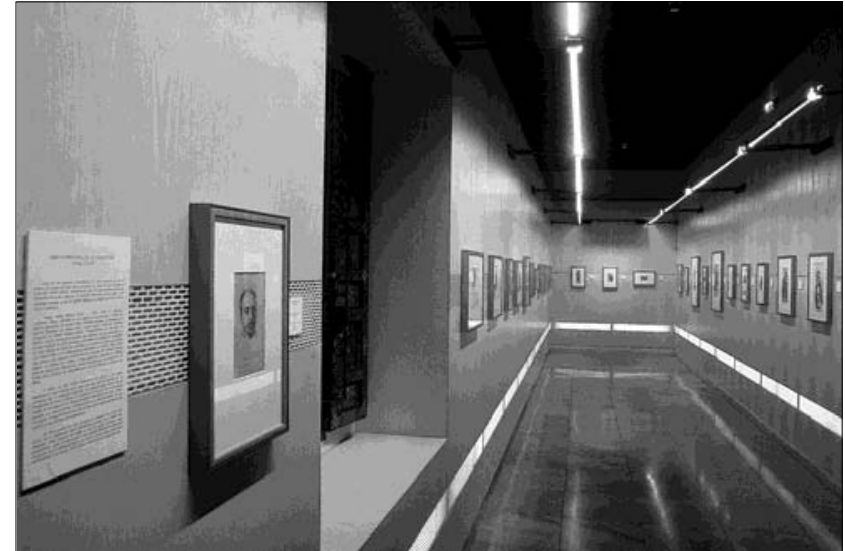

Además este sistema nos permite poder exponer las obras con todas las máximas garantías, previniendo el efecto invernadero que se puede producir también con la presencia de pequeñas cantidades de energía causando en los materiales higroscópicos una rápida e intensa deshumidificación, que comportaría en el caso de las obras sobre papel, una contracción de las fibras celulósicas del soporte a menudo irreversible.

3. La iluminación pensada para la nueva situación expositiva, se realizaría mediante el empleo de "Guías de luz a emisión prismática", un sofisticado tipo de conductor óptico expresamente puesto a punto para la iluminación de tejidos, tapices y documentos gráficos sobre papel.

Describiremos a continuación el Proyecto llevado a cabo en la confianza que sirva de ayuda a otros colegas para mejorar los sistemas expositivos de obras sobre papel tanto en exposiciones temporales como permanentes, incidiendo de manera fundamental en aquellos aspectos directamente relacionados con la conservación como base de la difusión de estos bienes culturales ${ }^{8}$.

\section{Diseño del espacio expositivo y de los ele- mentos de montaje}

El espacio prexistente, de $14,50 \times 3,20 \mathrm{~m}$, condicionaba el proyecto museográfico, ya que en el convergen las entradas a la biblioteca y administración y la sala de arte cordobés medieval y renacentista, junto al acceso directo a la escalera del edificio. Además había que contar con la existencia de seis ventanas y una hornacina protegida con unas puertas de madera y otras de cristal, vaciada en el muro de fachada y en la que se expone un Crucificado de marfil.

A estos condicionantes se añade otro de importancia, marcado como en el resto del museo, por la falta de espacio.

Decidida la eliminación de las vitrinas preexistentes y elegida la opción de exposición directa sobre los paramentos verticales de la sala, se planteó la evidente necesidad de rentabilizar al máximo el espacio disponible, 
$\frac{1}{0}$
$\square$



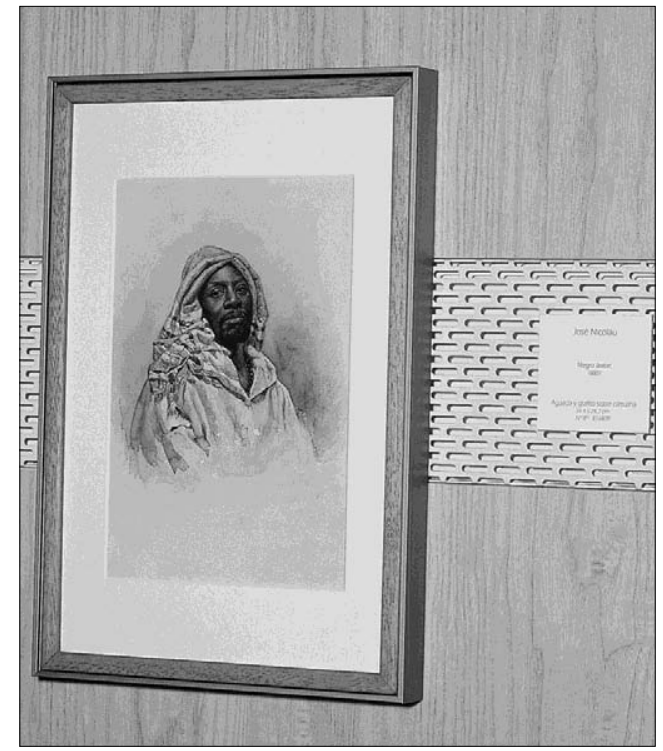

diseñando un sistema de paneles de madera que por un lado aislaba los dibujos y grabados del muro y por otro, aumentaba el espacio disponible para exposición. Se diseñó, así, un recubrimiento perimetral del espacio, revistiéndolo de un falso muro de contrachapado en terminación de madera clara, de poca beta y protegida por un barniz mate. La altura del nuevo muro de madera se trazó a 3,10 m, diseñando tanto al principio como al final de la sala, un saliente de 20 $\mathrm{cm}$ de ancho por 40 de profundidad perpendicular al plano expositivo.

Tras este muro perimetral se diseñó un pasillo técnico, que permite a través de un elemento móvil el acceso a cinco de las seis ventanas de la sala, la instalación de las conducciones eléctricas y la previsión de instalación de las conducciones de los sistemas de climatización actualmente en proyecto.

Para la hornacina en la que se expone la escultura se realizó un cajeado en la panelación, creando dos planos diferentes. Uno, el principal, que continúa el nivel del plano expositivo en el que se cuelgan los marcos y otro, el secundario, que abarca la hornacina, todo ello con la idea de dar continuidad y unidad a la sala.

Embutidos en estos paneles se diseñó un sistema de enganche de los marcos en los que se exponen dibujos y grabados que se fijan sobre una rejilla metálica perforada, cuyo eje central está situado a I,50 m del suelo. La rejilla va pintada en el mismo tono de la madera de panelación y a ella se fijan los marcos mediante unos tornillos diseñados a tal efecto, usándose también como soporte de la rotulación de las obras expuestas mediante un sistema de imanes.

\section{Los marcos como elementos de conserva- ción y seguridad}

Los marcos usados para este nuevo montaje fueron diseñados para la exposición Dibujos del Museo de Bellas Artes de Córdoba, celebrada en los Museos de Bellas Artes de Córdoba, Sevilla y Granada entre diciembre de 1997 y octubre de 1998. El carácter itinerante con que esta muestra fue concebida marcó de forma definitiva el diseño de los marcos, una vez desechada la idea de mostrar los dibujos en vitrinas cerradas. Primordial en la concepción de los mismos resultaba tener en cuenta que los procesos de manipulación, embalaje, transporte y montaje debería repetirse en todas las sedes de la exposición, que en principio estaba prevista que itinerara por las ocho capitales andaluzas, aunque su itinerancia fue posteriormente reducida. Debían, por tanto adaptarse el diseño y construcción de estos marcos a una serie de medidas preventivas que, garantizaran al máximo la conservación y seguridad de los dibujos a exponer.

Para ello, y tomando como base el conocimiento de algunos otros marcos para exposición de dibujos y 
grabados de diferentes museos europeos, comenzamos a considerar cuales eran los elementos que deberían necesariamente estar presentes, para evitar -en la medida de lo posible- los daños que pudieran ocasionarse por la acción de las condiciones mediambientales, manipulación, embalaje, transporte, etc.

Decidida la elección de unos dobles marcos de seguridad, se establecieron las condiciones mínimas requeridas para los mismos:

I. Estabilidad en los parámetros climáticos, que debía conseguirse mediante el uso de materiales estables y que actuaran de barrera, contemplándose la posibilidad de incluir un material tampón en la parte trasera.

2. Separación en dos marcos independientes con una fijación interior entre ellos, lo que suponía un elemento añadido de seguridad, ya que el marco $A$ es el que soporta mayor intervención y manipulación, pudiéndose montar toda la exposición con estos marcos y una vez finalizado el proceso de montaje colocar el marco $B$ que es el que conserva la obra original.

3. Rápida identificación del marco de cada dibujo para el proceso de montaje, al llevar el marco A sobre su panel trasero una reproducción de cada uno de los dibujos a exponer.

Los marcos albergarían en su interior el dibujo, grabado o cualquier otra obra sobre papel, montada sobre un paspartú de ventana y $\mathrm{pH}$ neutro.

Estos marcos se están usando en el museo desde su diseño en 1997 y en ellos se trasladan y exponen las obras sobre papel a las exposiciones temporales fuera del museo, facilitando el montaje de los mismos y reforzando, como se ha visto, la seguridad de las obras expuestas.

\section{La iluminación. Criterios de elección de las fuentes luminosas}

A la hora de seleccionar las fuentes para iluminar un determinado bien cultural es necesario tener en cuenta una serie de consideraciones que nos determinarán los criterios de selección que emplearemos.

En primer lugar, sabemos que las radiaciones responsables de las degradaciones de los objetos que estudiamos no son todas visibles, ni están presentes en la misma proporción, sino todo lo contrario, varían sustancialmente según el tipo de fuente de luz seleccionada.

En segundo lugar, conocemos que el estudio de la composición de la radiación emitida por la fuente luminosa es básico para controlar la incidencia nociva sobre los bienes culturales. Por ello es conveniente saber que no es suficiente controlar, en el sistema de iluminación seleccionado, la ausencia de las radiaciones mas peligrosas, sino también, y aún si cabe más importante, verificar que la fuente luminosa no sobrepase los límites de iluminación máximos aconsejados para un determinado objeto.

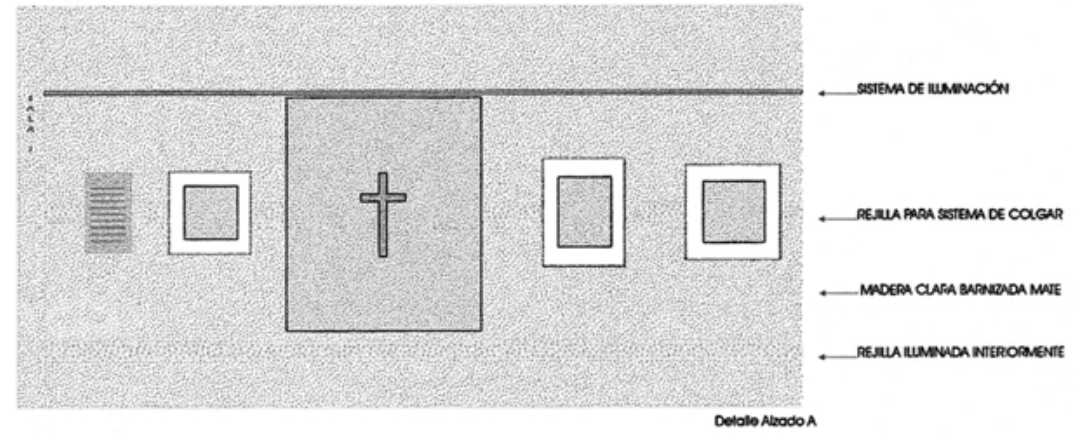

Propuesta del nuevo sistema expositivo

$\longleftarrow$
$\square$
Según un orden de prioridad los criterios de selección de las fuentes luminosas a emplear en la iluminación de bienes culturales son:

- La temperatura de color $\left({ }^{\circ} \mathrm{K}\right)$ y la distribución espectral de la fuente luminosa, sobre todo la longitud de onda de las radiaciones ultravioletas y de las radiaciones infrarrojas.

- La vida media, es decir las horas totales de funcionamiento, de las lámparas utilizadas.

- La eficiencia luminosa ( $\mathrm{m} / \mathrm{W}$ ) de las lámparas utilizada a fin de evaluar el consumo energético y las cargas térmicas introducidas en el ambiente.

Asumidos estas premisas, optamos por la elección de un novedoso sistema a base de Guías de luz a emisión prismática, diseñado en paralelo a la parte superior del plano expositivo, mientras que en la parte inferior del mismo se embutió una rejilla con iluminación fluorescente en su interior con la finalidad de iluminar toda esta zona.

\section{Iluminación y luminotecnia}

Los nuevos sistemas de iluminación estudiados y evaluados para esto proyecto nos han mostrado la existencia de un amplio abanico de posibilidades y de soluciones, pues el desarrollo tecnológico pone en el mercado toda una serie de fuentes luminosas, bien modificando y mejorando las existentes o bien inventando nuevos sistemas, siempre desde la óptica de su aplicación al campo de los bienes culturales, haciendo compatible su exposición y presentación al público con su conservación.

Entre los sistemas analizados figuran:

Las fuentes de descarga (fluorescente) son las más empleadas en la iluminación de bienes culturales, pero también son las más nocivas por la radiaciones de



UV que emiten. La acción de estas radiaciones potencian los cambios cromáticos en los pigmentos (palidecimiento) y favorecen la oxidación de los aglutinantes que se vuelven vidriosos y se separan.

Por ello si se quiere utilizar este tipo de lámparas hay que tener la precaución de filtrar siempre la luz emitida por ellas.

Hoy día ya es posible la regulación del flujo de la fluorescencia. Ya no es un problema poder hacer una iluminación de vitrinas regulando el flujo luminoso entre un 75 y un $90 \%$ según los casos. Además aportando las siguientes ventajas adicionales: disminución del calor disipado, mejor reproducción cromática de las fuentes, utilizando balastro de alta frecuencia, y emisiones de frecuencias que impiden el desarrollo de un posible ataque microbiológico.

La incandescencia clásica, ofrece una buena calidad de luz, resultando más cálida y acogedora. Permite distinguir bien los colores pero tienen el inconveniente de su menor duración en comparación con las fluorescentes y de un elevado consumo de energía.

Por su tipología emite rayos infrarrojos que recalientan los objetos y pueden alterar el microclima circundante dañando las obras.

Además de los sistemas clásicos de iluminación se investigó también en el campo de las nuevas tipología de iluminación por conducción.

Desde hace algunos años se ha empezado a utilizar unos nuevos métodos para la conducción y distribución de la energía luminosa por medio de sistemas que utilizan las características físicas de algunos materiales. Estos sistemas vienen denominados Guías de Luz.

El mercado luminotécnico en este momento produce diferentes tipos de guías de luz:

- De emisión longitudinal, que puede ser de dos tipos, la guía de luz hueca y la fluida., utilizando ambas el principio de la difusión óptica, pero es muy difícil obtener con ellas, un haz de luz direccionable.

- Sistemas de transportes y de emisión a través de fibras ópticas (fuentes puntiformes). Estos sistemas permiten una gran maniobrabilidad, pero tienen el inconveniente de no conseguir transportar grandes flujos luminosos. El ángulo de emisión es concentrado y por consiguiente, para obtener una uniformidad aceptable, es necesario realizar expositores o vitrinas de grandes dimensiones con el empleo de muchas fibras ópticas que, al iluminar objetos tridimensionales, produce múltiples y fastidiosas sombras, sin poder obtener en ningún caso una iluminación uniforme.

Como se puede observar, hoy día disponemos de una mayor variedad de sistemas de fuentes luminosas, pero decidir entre uno u otro es complejo, ya que si bien permiten solucionar algunos problemas, también acentúan otros. 
La iluminación pensada para la nueva museografía de la sala, ha sido realizada mediante el empleo de Guías de luz a emisión prismática, un sofisticado tipo de conductor óptico expresamente puesto a punto para la iluminación de tejidos, tapices y material gráfico sobre papel.

El conocimiento de la problemática luminotécnica y el estudio pormenorizado de las exigencias conservativas, ha permitido poner a punto un sistema que representa hoy día la respuesta concreta a tales exigencias. La experimentación de este nuevo sistema ha sido realizada por primera vez en la iluminación del Museo-Sacristía de la Capilla Real de Granada, usándose también para la iluminación de pinturas murales, de dibujos, de pintura, mosaicos en diferentes museos italianos en Roma, Asís o Reggio Calabria.

Este trabajo de estudio, investigación, y experimentación se ha podido realizar gracias al trabajo interdisciplinar entre especialistas de diferentes sectores e instituciones: luminotecnia, control del medio ambiente, conservación preventiva, arquitectónico, y por ultimo en estrecha colaboración con un laboratorio de ingeniería y construcción de los aparatos luminosos?. Gracias a esta colaboración ha sido posible poner a punto el sistema según un estricto protocolo científico correlacionado con las exigencias de conservación preventiva de los bienes culturales, obteniendo un producto en grado de garantizar las ventajas que exponemos a continuación:

- Solucionar las exigencias de iluminación y conservación en cuanto que se elimina completamente la radiación ultravioleta (mecanismo de degradación imputable a la luz) y se reduce la carga térmica en más de un 90\% (debido al hecho que el material utilizado es un polímero acrílico que no transmite calor por convección interna) transformándola en una fuente prácticamente fría, y en consecuencia, disponer de la posibilidad de someter a los bienes culturales a unos niveles de iluminación superiores a los normalizados a nivel internacional sin ocasionar daños en ellos.

- Anular la posibilidad de deslumbramiento ya sea primario o secundario ${ }^{10}$ gracias a la posibilidad de controlar el ángulo de emisión, lo que permitirá iluminar sólo al objeto que nos interesa y no al observador.

- Poder confinar la fuente luminosa en el exterior del ambiente expositivo de las vitrinas con un rendimiento superior a los otros sistemas de iluminación hasta hoy utilizados.

- Y por ultimo, la utilización de fuentes luminosas halógenas frías con lámparas dicroicas que permite eliminar hasta 2/3 de la energía total irradiada, con esto se evitará no sólo la introducción de calor en el interior de vitrinas, sino también la eliminación del fenómeno de la deshumidificación localizada, que podría resultar dañina para algunos materiales que necesitan un equilibrio termo higrométrico perfectamente estable.

\section{Guía de luz prismática: principios de funcionamiento}

El principio de funcionamiento de este sistema de iluminación está basado en el transporte de la energía luminosa a través de un material ópticamente estable (PMMA, polimetilmetacrilato) o cristal acrílico colado que presente el mayor índice de refracción posible y que tiene una curva de transmisión lineal en el espectro visible a fin de poder utilizar el fenómeno de la reflexión interna total, obteniendo de esta forma unos ángulos de emisión elevados y una gran eficiencia luminosa.

La emisión de la luz se produce a través de unos microprismas dispuestos en sentido longitudinal en la parte opuesta a la superficie de emisión, con una extensión proporcional al ángulo fotométrico que se desea obtener. Estos microprismas están impresos a control numérico con ángulos controlados so-



Sistema de montaje a pared y detalles constructivos de la guía de luz a emisión prismática

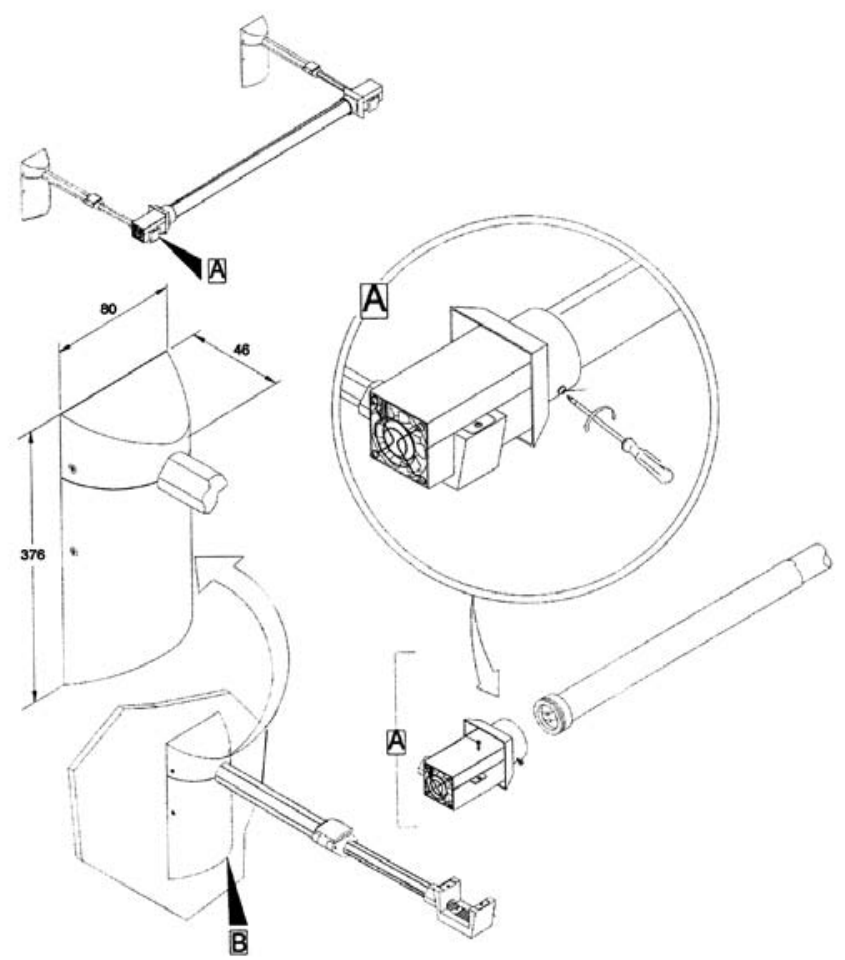



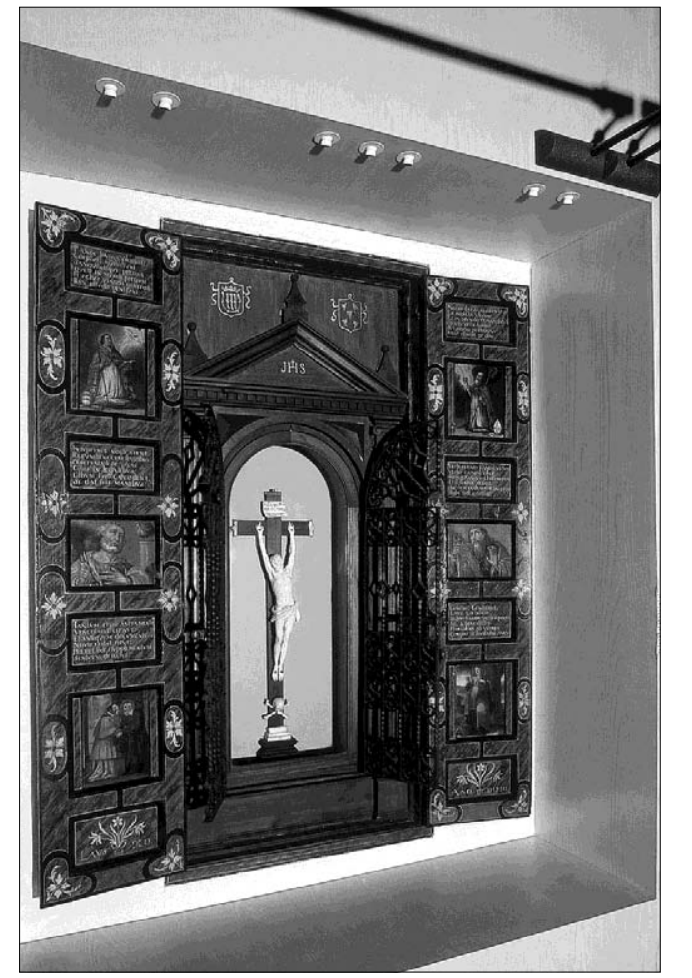

bre la superficie contraria a la de reflexión. Basándose en el principio de realizar ángulos tales que la reflexión interna total (caso particular del fenómeno de la refracción) permita la emisión de una parte del flujo luminoso sobre un eje perpendicular a la guía misma.

Este principio permite adquirir una mayor eficiencia de la que se obtendría introduciendo una superficie difusora en el interior del recorrido óptico (guía prismática hueca). Al igual que produce un haz de luz controlable en el ángulo de abertura tanto en sentido longitudinal como en transversal.

La luz visible transmitida presenta la misma curva espectrofotométrica que aquella introducida, o sea no existe aberración cromática en cuanto que el medio utilizado está constituido por metacrilato colado que tiene una curva de transmisión lineal en el campo visible, al igual que anula los componentes de ultravioleta y de infrarrojo lo que significa que no existe contaminación cromática por parte de la fuente luminosa, que mantendrá una emisión espectral invariada.

La utilización de este sistema sobre superficies pictóricas, permite obtener una optima uniformidad de iluminación, eliminando las reflexiones superficiales de los pigmentos, obteniendo en virtud del delicado equilibrio de refracciones internas entre barniz y películas pictórica, una exaltación del contraste general y en consecuencia una mejor visión del conjunto de la obra.

La flexibilidad de construcción deriva del hecho de "diseñar" los prismas en función del objeto a iluminar. Además este sistema de iluminación tiene la posibilidad de curvar las guías, los cual aumenta su campo de empleo: acentuar arquitecturas, iluminar fuentes, ya que es posible sumergirlas en agua, o dado que es un sistema de transmisión independiente de la fuente de iluminación utilizada, permite captar, canalizar y distribuir luz solar, para utilizarla como fuente de iluminación en interiores.

Podemos afirmar, sin miedo a ser desmentido, que el compromiso entre el disfrute visual del publico y los problemas conservativos es un hecho factible. No necesitaremos mas recurrir a una realidad virtual falsa y subjetiva para observar una obra de arte, cuando podemos tener la oportunidad de verla en la realidad, despreocupándonos de los problemas de conservación, ya que éstos estarán plenamente solucionados.

\section{Incidencias de otros tipos de iluminación en} la sala

Resueltos los problemas de iluminación de las obras sobre papel, había que solucionar la incidencia en las mismas de otros tipos de iluminación en su entorno que podían afectarle.

Así, para la hornacina del Crucificado se optó por la iluminación con fibra óptica. Para la escalera, decorada con pinturas murales, se eligieron bañadores de pared colocados en una estructura octogonal que repetía el módulo del motivo central del artesonado iluminado mediante la reflexión de los bañadores y fluorescentes dentro del carril electrificado todo ello de Erco. La ventana de la escalera y la ubicada en el pequeño espacio de distribución de los diferentes accesos, y cuya iluminación natural repercutía directamente sobre la exposición, se protegieron con unos filtros constituidos por la superposición de diversas láminas de protección solar y ahorro energético (tipo Solar-check, de la firma DTI).

La conservación de un objeto, de una colección o de un monumento, es el objetivo más importante a tener en consideración, no sólo por los responsables encargados de su custodia y conservación, sino también por parte del público en general, no podemos olvidar que es deber de todos, cuidar, mantener y transmitir a las generaciones futuras el legado patrimonial que nos ha sido consignado.

En síntesis, los profesionales de la conservación están obligados a planificar y aplicar un programa de conservación y de restauración que proteja, con los medios técnicos y humanos adecuados, los bienes culturales contra todo proceso de destrucción o de alteración. Ello requiere un conocimiento profundo de los agentes de deterioro externos e internos y del comportamiento y reacción de la propia naturaleza material de los objetos a las agresiones del entorno, para poder aplicar la metodología preventiva y curativa más efectiva y respetuosa posible, sin perder de vista la realidad poniendo en practica el más simple, pero al mismo tiempo el más complejo de los sentidos, que es el sentido común. 


\section{Bibliografía}

ARAMINI, F. (1992) lluminación y estudios colorimétricos aplicados a la exposición del bien cultural. En Un proyecto para la CapiIla Real de Granada. Serie Cuadernos n I, Cádiz, pp. 77-80.

A.A.V.V. (1985) Vitrines. Museum, $n^{\circ}$ I46. Paris.

BAGLIONI, R. (1996) Vitrina de conservación del esmalte denominado "Tríptico del Gran Capitan" del Museo de Bellas Artes de Granada. Proyecto y realización. En PH Boletín del Instituto Andaluz del Patrimonio Historico, No I5, año IV, Junio 1996, pp. 75-82.

BAGLIONI, R. ( 1997) Proyecto de sistema expositivo para la Bandera de las Navas de Tolosa, Vilches, Jaen. Informe interno del IAPH Enero 1997.

BACCAREDDA BOY, M. (1976) Materie plastiche ed elastomeri. Quaderni di chimica applicata, a cura di E. Mariani. ED. Ambrosiana 1976, pp. 156-167.

BERDUCOU, M.C. (1990) La conservation en Archéologie. Ed. Masson.

CASTELLANO, Ma T. - ESPEJO, P. (1983) Grabados pertenecientes a la Calcografía Nacional en el Museo de Bellas Artes de Córdoba. Los hermanos Manuel y Juan Antonio Salvador Carmona. En Apotheca. Revista del Departamento de Historia del Arte, Universidad de Córdoba, n³, 1983, pp. 21-35.

CHAIMOWICZ J. (1990) Ondas luminosas, introducción a la tecnología optoelectrónica. Ed. Paraninfo. Madrid.

DEMENY, L. (1967) Degradation of cotton yarns by light from fluorescent lamps", 1967 London Conference on Museum Climatology (ed.G.Thomson). IIC Revised Ed. (1968) pp. 53-64.

EGERTON, G.S. - MORGAN, A.G. (1970) The photochemestry of dyes, partsI and 2. Soc. Dyers colour, pp. 79-83 y 242-249.

GARCÍA DE LA TORRE, F. (1997) Dibujos del Museo de Bellas Artes de Córdoba. Catálogo de la exposición. Museos de Bellas Artes de Córdoba, Sevilla y Granada, diciembre 1997- diciembre 1998. Sevilla.

GARCÍA DE LA TORRE, F. (2000) Dibujos y grabados en el Museo de Bellas Artes de Córdoba: Historiografía, museología, museografía y conservación. En Actas del Encuentro Internacional sobre Conservación del Patrimonio Documental y Bibliográfico en clima subtropical. Santa Cruz de la Palma, 19-25 de julio de 1999. Cuenca, pp. 5 I-70.

GARCÍA DE LA TORRE, F. (200I,a) Colecciones sobre papel en el Museo de Bellas Artes de Córdoba. En Actas del IV Congreso Nacional de Historia del papel en España. Córdoba, 28-30 de junio de 200I. Cuenca, pp. 15-31.

GARCÍA DE LA TORRE, F. (200 I,b) Homenaje a Alonso Cano. Dibujos. Catálogo de la exposición. Museos de Bellas Artes de Córdoba, marzo-septiembre, 200 I y Sevilla, octubre-noviembre, 200I. Córdoba.

GARCÍA DE LA TORRE, F. ( 2002): Museo de Bellas Artes de Córdoba. A media luz. La sala de dibujos y grabados. Mus-a Revista de las instituciones del patrimonio histórico, año I, nº 0, 2002, pp. 32-37.

GAYA NUÑO, J.A. (1968) Historia y guía de los museos de España. Madrid, 1968.

GAYA NUÑO, J.A. (1981). Vida de Acisclo Antonio Palomino. Córdoba.

GUICHEN, G. de (1984). Climat dans le Musée. ICCROM. Roma.
HARRIS, M. - SMITH, A.L. (1938) Photochemical reactions of wool. En J. Res. Nat. Bur. Stand 20 (1938), pp. 563-569.

HARRIS, M. (1934) Photochemical decomposition of silk. En Am. Dyestuff Reptr. 23 (1934), pp. 403-405.

HECHT, A. Zajac. (1986) Optica. Ed. Addison.

HERRAEZ, J.A. - RODRÍGUEZ LORITE, M.A. (1989) Manual para el uso de aparatos y toma de datos de las condiciones ambientales en museo. Ministerio de Cultura. Madrid.

HERRAEZ, J.A. - RODRÍGUEZ LORITE, M.A. (199|) Recomendaciones para el control de las condiciones ambientales en exposiciones temporales. Instituto de Conservación y Restauración de Bienes Culturales. Ministerio de Cultura. Madrid.

ICOM, ( 197I) La lumier et la Protection des objets et Specimenes exposés dans les Musées et Galeries d`Art. L’Association Francaise de l'Eclairage, s/f. También en Lux, 63 (197I), pp. 235-264.

ICCROM, (1975) Catalogues of Technical Exhibitions, No I-Lighting. Roma.

LITTLE, A.H. (1965) Deterioration of textile materials. Delft Conference on the Conservation of Textiles, IIC (2nd Ed. 1965), pp. 67-68.

PADFIELD, T. - LANDI, S. (1966) Light-fastness of de natural dyes. En SIC II (1966), pp. I81-196.

PADFIELD, T. (1969) Deterioration of cellulose, a literature review. En Problems of conservation in museums. ICOM (1969), pp. 119-164.

PALENCIA CEREZO, J. Mª ( 1999) Velázquez grabado. Catálogo de la exposición. Museo de Bellas Artes de Córdoba, junio-octubre, Córdoba, 1999.

PAULILLI, G. (1995) L' illuminazione ecologica. En Media Production, $n^{\circ}$ 64. 1995, pp. 16-21.

RADI, P. ( | 993) || sistema d' iluminazione a Guide di Luce. En Luce, anno 32, n², marzo/aprile. 1993, pp. 68-72.

RODRÍGUEZ LASO, Ma D. (1999) El soporte de papel y sus técnicas. Degradación y conservación preventiva. Bilbao.

RODRÍGUEZ LORITE, M.A. (1994) La luz en el Museo. En Miscelanea Museológica. Servicio Editorial Universidad del País Vasco, pp. 229-24.

SAECHTLING, H. (1986) Manuale delle materie plastiche $3^{\text {a }}$ edizione. Tecniche Nuove, Milano.

STOLOW, N. (1987) Conservation and Exhibitions. Butterworths.

THOMSON, G. (1986) The Museum Environment. Second Edition (1986). Ed. Butterworth-Heinemann. Edc. Española: El museo y su entorno, Madrid, 1998.

VAN BEEK, H.C.A. - HEERTJES, P.M. (1966) Fading by light of organic dyes on textiles and other materials. En SIC II (1966), pp. 123-132.

VANNUCCHI, G. (1982) Ottica integrata e microottica, vol I y 2. Ed. Pátron.

VILLAVECCHIA-EIGERMAN (1975) Nuovo dizionario di merceologia e chimica applicata,. Milano, pp. 2528-2529.

Fotografías: Museo de Bellas Artes de Córdoba. Álvaro Holgado. Córdoba

\section{Notas}

I. Para un estudio más completo de la colección de dibujos véase: García de la Torre, 1997, 2000, 200 I- a, 2001 -b y 2002.

Igualmente, para la colección de grabados véase: Castellano / Espejo, 1983, Palencia, 1999, García de la Torre, 2000, 200 I-a y 2002.

2. Archivo M.B.A.Có. Legajo 43, "Año económico de 1876 a 1877. Periodo ordinario" y Legajo 39 "Memoria que contiene las actas 


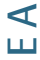

ш archivos y museos de titularidad estatal y sobre gestión de bibliotecas de titularidad estatal, estipulación 2a , 2.4., y de otras normas estatales y autonómicas, la Junta de Andalucía realiza el ingreso de sus fondos en los museos gestionados en calidad de depósito, independientemente del origen de su sistema de incremento como adquisición o donación.

4. Para más datos sobre el tema véase: García de la Torre, 1997. 2000, 2001-a y 2002.

5. Gaya Nuño, 1968, pp. 247-248 y 198I, p. 104.

6. Estas vitrinas fueron diseñadas y ejecutadas por la empresa Riobe, de Córdoba.

7. García de la Torre, 1997, p. 36.

8. La ejecución material de este proyecto fue realizada por: Francisco Rojas, Juan Ruiz Ayala, Francisco Bermón, Antonio
Ajenjo y Novolux, de Córdoba, Deluz, de Sevilla y Desisti Lighting, de Roma.

9. Este trabajo de investigación ha sido posible gracias a la colaboración de los técnicos del laboratorio del Física del Instituto Centrale del Restauro de Roma, Fabio Aramini y Carlo Cacace, al arquitecto del Proyecto museográfico de la Capilla Real de Granada, Pedro Salmerón Escobar, al conservador-restaurador del Instituto Andaluz del Patrimonio Histórico Raniero Baglioni y la empresa de iluminación Desisti Lighting de Roma.

10. Se entiende por deslumbramiento primario el derivado de la propia fuente luminosa, mientras que por deslumbramiento secundario se entiende el producido por la reflexión de la fuente luminosa por parte del objeto iluminado. 